\title{
Notion of Strategic Control
}

\author{
Qemajl Sejdija \\ PhD Cand. European University of Tirana
}

\begin{abstract}
With strategic control, we understand the special type of strategic control which is aimed to monitoring and evaluation of strategic management process in order to ensure its functioning and progress. In fact, the strategic control is applied to ensure if all the planned results are accomplished during the process of strategic management. The basic purpose of strategic control is to assist senior management in achieving the objectives of the enterprise with the assistance of monitoring and evaluation of strategic management in general, and particularly in the implementation of the selected strategy. Relying on the preliminary explanation, the results of the assessments are reflected in the strategic management of the enterprise environment, the establishment of vision, mission and goals of the strategy and the conversion of strategy into shares. Strategic control in this case appears as a reverse link that provides feedback to determine whether all stages of the process of strategic management and how they function have been implemented in harmony.
\end{abstract}

Keywords: Notion of Strategic Control

\section{Introduction}

The stages of strategic control are closely linked among themselves while comprising a compacted integrity, in which each of these stages is of uniform significance and therefore with an equal impact on the success of the entire control system. Therefore, upon the designing process of this system in current terms, to each of these respective organizational stages an appropriate responsibility should be given, regardless of the fact that some of these steps will be shaped easier and some more difficult. In other words, the duration of the configuration of any phase of the process and the complexity of its processing must not affect the favouring, i.e. their discrimination, while taking into account that the success of the control system depends on the success of each stage of this system.

In the context of reviewing the model of strategic management process it is clear that control and evaluation give the feedback of the entire model. The emitted information through this itinerary are the outcome of the current collected performance measurements. If any avoidance occurs between the achieved standards and performance, then operational managers need to know how, in what way they can correct the activities of the employees. In this case the involvement of senior management is not necessary. However, if such deviations result of the standards themselves, both, senior management as well as other management levels needs to know how to develop new programs and implementation procedures. In other words, high levels of management need to establish new standards of performance that will be in harmony with existing circumstances.

\section{Process of control and its features}

\section{Determination of measuring sizes}

Determining of the measuring size means to measure what should be the starting point in shaping the control system. In other words, management must demonstrate what it wants to measure, i.e. what are the processes and outcomes, by measuring the accomplishment of which is understandable that a company is making its own strategy. The ability to solve these processes and results constitutes one of the basic skills of managers. Related to this following questions should be made:

1. What processes and results represent the best the intentions of the responsibility scope of the respective manager?

2. What is the failure of the responsibility goals of the respective manager manifested with? 
3. What is the best measure of critical accomplishment deviation from the goal?

4. In what way it can be learned who is responsible for the respective error?

5. Which indicators of goals development will cause the least cost?

6. For which indicators can be obtained information in economic terms?

Resolved processes and results should be measured through appropriate measurement and in rational way. Thus, the most important elements of the process are placed in the centre and the elements that have the greatest impact on costs or those that cause the biggest problems in the enterprise.

When defining the performance of the goals, which will be measured in the process control, it is usually initiated by the aspirations of all stakeholders, such as owners, managers, employees, buyers, suppliers, banks, government and others.

\section{Setting of standards}

Standards represent the size of goals by which is compared the current or expected effects (performance).In this sense, the standards are represented as measures or criteria under which it estimated the accomplishment of the goals and tasks of individuals, workgroups, and organizing units and the enterprise as a whole. If the standards are set properly, from the early development process of any activity they signal that this process is being developed within or outside the planned framework. In this case they also signal the manager to take certain actions, which will return the process of such planned framework, in cases when deviations are outside the limits of tolerance.

Standards can be of quantitative and qualitative character; the first ones are measurable, while the latter ones are appreciated.

Quantitative standards usually appear as physical (neutral) and valuable standards, whereas, quality standards appear as descriptive, programmatic and targeting.

Physical standards are neutral sizes presented in absolute or relative way - kilograms, tons, meters, ton- kilometres hours according to tons hours, according to the production unit, etc.

\section{Compare: Weichrich, Koontz, H. Op. ciut. page 581}

Standards with valuable are absolute or relative sizes presented in currency, and deal with expenses, (direct and indirect, special and general), expenses, incomes (general and specific), then capital (rate of return, the ratio between net debt and the ratio of the following assets and subsequent debt, etc.).

Descriptive standards deal with such measures which may be presented in a quantitative way. This primarily has to do with the features, activities and processes which can be evaluated later.

Program standards are of such nature that some activities from a certain program (eg program of new product acquisition), or even the whole program is treated as a standard.

Aimed standards are issued from set goals and deal with those purposes that are not of quantitative character (eg increase the level of knowledge or ability, creating better working brightness).

Each of these standards will be applied in this case depends on the type of activity which is developed by the manager. In an enterprise, according to the set rules all types mentioned standards will be used.

\section{Measurement of current performance}

Measurement of current performance a procedure in which is determined if the set standards have been accomplished and to what degree they have been accomplished, then the reasons are determined why possible deviations have happened to achieve the same, it is necessary to provide the relevant information regarding what has been accomplished and has failed to accomplish in terms of the enterprise as a whole, the said information has to deal with the marketing and sales, production, supply and stocks of materials, capacities, defects and maintenance, workforce, involved capital and the success of the business. Collection and processing of this information can be performed by the manager himself, 
appropriate service which is relevant to the manager or a separate service outside the jurisdiction of the relevant manager information systems based on electronic computers that are designed for these purposes (EIS, MIS)T are particularly effective.

To ensure the effectiveness of performance of measurement, collection and processing of information must have a certain dynamic-certain information will be collected during the day, others within a week or 10 days, some within a month, and some even within 3 months, half a year or a year. Processing of such information is done in a way to be comparable with set standards, in order to make the possible deviation visible.

In terms of the possibility of influencing the accomplished results if possible, it is the best that during their period of their accomplishment to collect information about deviations in order to act preventively. More often here we have to deal with the costs and expenses that about to be made for any final accomplishment. In this case the standards are set for each part of the final performance, so the implementation process signals that are part of this implementation present avoidance of costs and expenses, in order to take preventive actions prior to the end of the whole process. On the analogue way the measurement of performance is set with respect to the implementation of the investment program, implementation of which is followed according to the stages of development.

\section{Comparison of current performance standards}

Comparison of performance and standards is a procedure in which a comparison of different data on these performances and performance standards is done. The purpose of this comparison is to determine:

1. Avoidance of performance standards,

2. The sizes of deviations from standards,

3. The causes of deviations from standards.

In cases where the current performances sizes are within the desired tolerance, measurement process stops here. However, if the deviations are outside the tolerance limits, it is necessary to first prove their pre-signals. In this way, we should note that avoidance from standards can be negative and positive. Not only negative avoidance signal for action, but action signals can be both positive deviations, thus any avoidance from standards must be subjected to detailed analysis. This analysis should also provide answers to the question of why the avoidance has happened -was it conditioned to the subjective or objective reasons.

As the comparison of performance standards with determining of the size and direction itself (pre-signal) avoidance can be performed automatically, the determining of the avoiding cause is a matter of special analysis. Such analysis, in certain cases is carried out by the manager, whereas in other cases, they should be carried out by a special team of experts. It can be said that at the operational level the standards are usually of common size, and therefore the caused avoidance can be verified more easily. Despite this, at the strategic level the standards comprise complex accomplished s sizes that are subjected to numerous influenced factors. Therefore, the determination of the causes of avoidance in such cases is a complicated process which requires long and detailed analysis.

\section{Evaluation of performance and corrective actions}

Evaluation of performance means analytical and diagnostic means of the manager's ability, regardless whether if it develops the analysis of the causes of avoidance or is carried out by specialized service. Without this ability the manager would be handicapped upon decision-taking on actions that should be taken to eliminate or soften such avoidance. Most often three following aspects of these actions are distinguished:

\section{Maintaining of the status quo}

2. Correction of avoidance

3. Change of standards

The maintaining of the status quo is recommended in cases where deviations from the standards are so small and it's not worth to undertake any actions that would ensure the implementation of standards. However, when the avoidances are 
large, then the correction of avoidance is indispensable. If such actions aren't taken than major disturbances will take place in the enterprise business. What corrections will be taken depends on the causes that have contributed to the avoidances from the set standards, and it is determined by analysis. Considering it from the functional point of view, such causes can be identified in planning, organizing, and management of human resources -i.e. with functions of management,

\section{Griffin, W. R. , Management, 3rd Edition, Houghton Miffin Company, Boston, 1990, page 606.}

that precedes the control. This means that corrections can be made in the plans and purposes, organizing and determination of the staff and the style of leadership itself. This shows that the control is not only an integral feature of the management of the main action, but also retroactive for all previous functions.

The change of standards is usually a consequence as they are unrealistically set, too high or too low. Very high set standards, are usually diagnosed when with those whom we have to do, they are not capable to accomplish. In contrast, very low set standards are diagnosed when most are implemented very easily but fail too high.

All this indicates that the evaluation of performance in selection of appropriate management action is fair.

\section{Types of control systems}

The enterprise acts (operates) in certain environments and by its existence depends upon its reception. Therefore, it must control how the environment reacts to its outputs. On this occasion we constantly have to bear in mind such outputs are the result of the internal operation of the company (the process of transformation or conversion), therefore, from such an action depends the solvency of outputs, and that means its acceptance them from the environment. All this shows that the determining of the success of the chosen strategy should control two basic sets of performance-a group comprised of performances of which mediation the company defines its accession (corrections) in the environment, whereas the other group consists of performances that define the ability of the enterprise for acceptance. Therefore, there are two basic types of control system:

\section{Out-organized system control,}

2. Organizational system control.

That both systems present a reality of each enterprise - the fundamental question is how are they formed and implemented. In this case we must consider that the control systems are very expensive, but they are often the only means of the enterprise to monitor and evaluate the performance in cases when organizational activities are complex.

\section{Out-organizational system control}

As the name itself indicates, this control system is operating outside the enterprise, and is comprised of market mechanisms that through the action of the offer and demand shows if the enterprise has been accepted and to what extension of the environment. This mechanism is most commonly called market control; implemented through the establishment of a system of prices, followed by mediation and the performance of the enterprise is evaluated. This is the objective of the control outputs. It is possible only when the enterprise is able to impose objective financial measures of performance.

There are three general forms of market control:

1. The market price of shares

2. The rate of return of capital,

3. Transfer of prices.

Out-organizational system control can work out successfully only if an adequate system of comparison is set In this case the system of comparison should first of practices should include the so-called special comparison, which means individual comparison of enterprise with competitors, in groups or branch of the activity.

The market price of the share. The share price is a performance measure of the company considering that it is the result of supply and demand in the market of capital. The changes imply reaction of the actors-starting from stock owners, managers of enterprises and to a range of external stakeholders-suppliers, buyers, banks, governments, etc. However, 
considering that we are dealing here with controlling the implementation of the chosen strategy of the enterprise, these implications are primarily related with the management of the company, which should adapt its behaviour to such changes. In fact, if the share prices have risen compared to their nominal value, this is a sign that management has chosen a real strategy, so it will still enjoy the support of shareholders. Otherwise, if the stock prices have fallen below the nominal value, this may be a sign that management hasn't chosen the true strategy; therefore, it will lose the support of shareholders. Particular sensibility of management occurs when the changes in stock prices reflect the level of management compensation. This happens not only when a part of the management of share profits is paid, but particularly when applying the system of values of options. This is such a form of compensation which is paid to the managers in shares under a fixed price, which in most cases is equal to their market price on the day of admission. Options are usually allowed primarily, and then later are allowed the General Assembly. Stimulating character of these options is whether the difference between the prices according to which the share is estimated for managers and the real price that will be achieved for such shares in the capital market, presents a motivation of successful enterprise business. Thus, in this case for managers it is very important that they choose and implement the strategy that will ensure continuous growth as the value stock, but it is a strategy that ensures the success of enterprise business.

\section{The rate of capital return}

This indicator shows the ability of the enterprise to the set deadline to return the capital to the investors. In this case the invested capital can be understood as the invested capital and used capital (total equity and long-term total shareholder) from which derive other different indicators such as, ROI, ROCE, ROA.

It is understandable that each strategy will not be equally successful - the more successful the strategy will be the strategy that provides the fastest return of the invested material. In other words, if the management has chosen

\section{Hill, G. R. , Jones, C. W. L. , Strategic Management - An Integrated Approach, Houghton Mifflin Company, Boston, 1989, page 264.}

a strategy that within a short period has enabled the return of their capital, this strategy is successful. For this reason, the height of the rate of return on the invested capital is the best indicator of the speed of this conversion and thus the success of the chosen strategy.

It should be noted that the rate of return of the capital is not only a measure of the performance of the enterprise, but it is also a measure of performance units of the divisions. In fact, even when the rate would to be used in comparison with the same enterprise or different groups and their subsidiaries, it will in deal the same way with comparisons to the same interdivisional enterprise. Thus, this is a very important measure of the performance of the enterprise and once the key control of the mechanism to the success of the chosen strategy.

\section{Transfer prices}

In multi-divisional enterprise, where relatively autonomous parts operate undertaking, (profit centres, business units, and strategic business units) the overall quality performance is expressed in the quality of each of these parts. Thus, the fundamental issue is how in the system of price as a mechanism of control that is outside of these parts, can control, check and evaluate the performance of the said parts. What we are dealing here with is the choosing of such price strategy in exchange with inter-divisional, which shall ensure the success of the enterprise as a whole. In this sense it is possible to distinguish two types of transfer prices - established market prices and established expendable prices.

The prices established in the market are those transfer prices in exchange with inter-divisional, which are determined based on market prices of internal transferred premises and these most often they are the prices of full market prices adjusted to market prices or contracted in basis of the market price.

Based expendable prices are in exchange with inter-divisional transfer prices which are determined based on the costs of internal transfer premises and these are mostly full costs, variable costs or fully increased costs.

Each of the mentioned systems of the price has its advantages and disadvantages, requiring a strengthened analysis of all the factors that affect their resolution. Therefore, it is great responsibility of management that within the overall strategy to 
choose what price strategy which will ensure adequate admissibility enterprise in extreme environment and appropriate recognition of its parts due to the internal environment.

\section{Organizational control system}

Unlike outside the organizational control system which is set off the units, by which it controls the success of the chosen strategy, organizational control system is located in the unit. It applies in all cases where there is no possibility to use outside organizational control, because objective standards cannot be placed, objective measurement and evaluation of performance control. Therefore, in all these cases it is difficult and expensive to develop, even when they do not have a sufficient budget to overhaul enterprise control of the enterprise goals, the manager must implement the organizational control.

There are two types of control -The first type of bureaucratic control and the second is the group control.

\section{Bureaucratic control}

Bureaucratic control is based system on the general rules and procedures for guiding the behaviour of stock or divisions, functions and individuals. These are not just impersonal rules and procedures, but also the budget rules and procedures and standardization of shares.

It is worth mentioning that this control system requires the active involvement of management, which here has the role of the instrument controller. In certain cases it is necessary to constitute a special body to control. All this has significant impact on the costs of the system and organizational control.

The rules of procedures Accomplishment of the chosen strategy is precisely conditioned with the certain behaviour of participants. Therefore, from the manager is expected to specify in advance which behaviours should be present, and this can be achieved with the issuance of rules and procedurave. ${ }^{4}$

1. The mode of action of future shares with procedures is determined in details; these are chronological sequences of implementing shares. With such is best described how the implementation of specific work routines and repetitive and are built for the enterprise as a whole and about its organizational units. The procedures are the basis of every part of the organization, so their absence would open the way to anarchy and thus the destruction of the enterprise.

2. The rules are specific requirements that do not allow freedom decision-making, which means that they are of directive character. They vary from the procedures, but are in the process, there are procedures that deal by any rule, but these rules are self-applicable. Therefore, rules and procedures are taken in those cases when the business of the enterprise is not desirable that the employees, respectively, executives of such rules and procedures, shall use free decision- taking.

3. The power of rules and procedures is that they standardize the behaviour of the employees. If the employees respect the rules set forth, actions or decisions will be implemented in the same manner and at the same time, so the result will be the expectation and accuracy, which is the purpose of the control system.

The budget is usually defined as a numerical overview of the outcomes. This transcript can be in the form of value (cash) or in neutral form. In fact, it is a collection of rules for the allocation of the resources, primarily financial ones.

There are three types of budget of classification, but all can be summarized in ${ }^{5}$ :

4. types of budget considering the budget period,

5. types of budget considering the inclusion of activity levels ,

6 t. types budget regardless to its content,

7. types of budget considering the methodology of research of budget sizes.

Buble, M. , op. cit. faqe $214-215$.

For more deatails see:: Buble, M. , op. cit. page 655-657. 
8. The period related to the budget may be different, but it should be careful to enable more precise evaluations and with best opportunities for implementation of the chosen strategy. Relaying on this aspect the annual budget and the capital budget usually changes.

a. The annual budget is the basic and the most important type of the budget, which as part of the strategic plan of the enterprise is transformed into an expression over a period of one year. Different from this, the capital budget includes precalculation of necessary capital investments for five to ten years.

9. Taking into account the activity of the enterprise the budget usually differs from its budget units.

a. The budget of the enterprise includes all activities of the enterprise as a whole and deals with long term or short term.

b. The parts of the enterprise covers only those parts of the budget of activity dealing with the relevant parts of the enterprise. The content of this budget depends on the type of organizational unit this budget deals with-whether it is strategic business unit, business unit, profit centre or just spending centre.

10. Taking into account the main budget usually is differs from particular special budgets that are directly or indirectly part of the main budget.

a. The main budget (Master Budget) usually consists of separate budgets connected between them, which in this total symbiosis represents the business activities of the future enterprise. Special budgets in this structure vary in content depending on the type and size of the company, however, including certain fixed contents.

b. In large enterprises, especially those multi-divisional, in addition to the main budget other budgets are constituted about each centre of responsibility. They are usually called responsibility budgets (Budget Responsibility).

11. Depending on the way they express the size, the budgets presented in the way of values and natural are usually the different.

a. The most common occurrence is the budget presented as a value, but any of the budgets of the budget follows the natural budget, it is the budget of working hours, budget machinery working hours, the budget of the quantity of products, etc.

b. Taking into account that the budget represents the state and the real state of certain sizes that have character of inputs or outputs, it enables dual control of the budget:

12. the budgetary control of the following activities,

13. budgetary control of performed activities.

14. Budgetary control of the following activities has a preventive control character, considering that it has to do with the ongoing activities. This means that it can be maintained within the framework of the planned activities. On the contrary it will signal the need for specific actions (corrective) which will provide a new alignment between separate budgets.

15. Budgetary control of the accomplished results is of correctional character, as presented after the activity is implementedit means this control is the output control. It is implemented by comparing the planned sizes and the executed ones, how the management will be able to:

16. determine the causes of these avoidances,

17. foresee and take appropriate measures,

18. localize the responsibility of such avoidances,

19. assess the performance.

In case of avoidance (deviations) between the planned and the implemented sizes ones it is possible to use the static and dynamic sizes that are formed in budget planning. Until the static access takes to compare the sizes of the budget, despite the volume of accomplished production (or sales), dynamic approach to control firstly the size of planned results in the volume of accomplished production (or sales), and then compares with real sizes. According to the last method the real result of expenses is, which enables an adequate action to be taken. 
For standardization it is possible to speak about the case when the content of the labour is specified or programmed. This means that in this direct way it affects the stand of the organization, with the aim of accomplishing the chosen strategy. It can be said that organizational control in the majority is based on standardization, since all the rules and procedures as well as budgets are based precisely on standardization. However, the rules are only a segment of standardization considering that it includes three aspects, as follows:

1. standardization of inputs,

2. standardization of throughputs,

3. standardization of outputs.

Standardization of inputs is a route how the organization can conduct human and material resources, even before engaging them in the process of work. Therefore, here we have to deal with so-called preliminary control (control Navigator motion control or retrospective), which is focused on the input of all resources in the enterprise. As for the human resources, this control is focused on recruiting and their selection and in accordance with the requirements of the work. In other words, in the process of human resource control will be accepted only those individuals whose performances are in line with the performance of the work.

In terms of material resources, this control is focused on the performance of materials such as quantity, quality, design, and trust, etc. Their behaviour in relation to the requirements of performance and on the basis of this decision-making whether, the inputs will be accepted or not accepted. In this way, the solvency of inputs is provided which is one of the assumptions that even further processes the processes in such inputs will be satisfactory.

Standardization of throughputs includes all activities pertaining to the transformation or conversion of inputs into outputs. Its task is to ensure that it takes place exactly as it is defined before

(solved in technological way or programmed) and activities to be developed in a manner and at the same time. Therefore, the desired goal of this standardization is predictable. Technological rules and procedures are key paths to achieve this standardization.

Control is selective (check yes/no and simultaneous control) and is focused on the process of transforming inputs into outputs.

Standardization of outputs has to do with what becomes specification of the characteristics of the performance of the product or final service (dimensions, tolerances etc.) in order to provide them the company applies the so-called after-action control, which is focused on the output or result of the enterprise, following the completion of the transformation process. For this purpose, the enterprise control systems use attributes such as QC, TQC and others.

\section{Control of the group}

Different from bureaucratic control, control of the group is no formal arrangement and structured organic view of the behaviour of individuals and groups in the enterprise, and is a characteristic of the organizational structures of authorities. The behaviour is by group standards with culture of the corporate culture and self-control. The system of services is not individual performances but in groups.

Different from bureaucratic control which is a mechanism that is outside the group, the control group is the mechanism that formed by the group itself by setting an internal system of values and organizational standards. Its purpose is self-control, when the individual feels a responsibility to work at his/her best, both for its own interests, as well as the for the interests of the enterprise. Therefore, the control group of employees are not controlled by any violent external system, such as direct superiors or separate control group. In this case, they create themselves their own system of standards and values according to which they behave.

\section{Conclusion}

Thus, with full responsibility we can say that values are relatively stable concepts, durable and fundamental in what they tend to achieve and strategies are specific types of operationalization. They constitute the basic framework for behaviour and leader. Important elements of successful enterprises are fundamental values such as quality, trust and service. The 
respect toward them in individual level presupposes the existence of the organization, reward, status, etc., but at the level of organization -successful implementation of the strategy.

Standards are concepts of desired behaviour forms, respectively standards for behaviour at work and the expected effects, by which the values are operational zed, respectively, the values are transformed into daily rules for the employees. It is believed that compliance of arranged standards is a condition for the existence and as well as the condition for profitability and success of the enterprise.

Values and standards are two important elements of organizational culture which is a part of each organization as a cause and consequence of the behaviour of individuals and groups in the organization. It is regarded as the most effective instrument of implementation of the strategy, therefore, without an adequate organizational culture, strategy simply cannot be implemented.

\section{LITERATURE}

[1] Buble, M., op.cit. page $214-215$.

[2] CompareE: Weihrich, Koontz,H. Op.cit. PAGE 581.

[3] For more details see: Buble, M., op. cit page $655-657$.

[4] Griffin, W.R., Management, 3rd Edition, Houghton Miffin Company, Boston, 1990, page 606.

[5] Hill, G.R., Jones, C.W.L., Strategic Management - An Integrated Approach, Houghton Mifflin Company, Bostor, 1989, faqe 264. 